\title{
DISEÑO DE PLATAFORMA MULTIMEDIA \\ PARA EL DESARROLLO DE LA COMPETENCIA \\ INTERCULTURAL COMO ESTRATEGIA DE \\ INTERNACIONALIZACIÓN EN LAS ORGANIZACIONES
}

\author{
MULTIMEDIA PLATFORM DESIGN FOR THE DEVELOPMENT OF INTERCULTURAL \\ COMPENTECE AS AN INTERNATIONALIZATION STRATEGY IN ORGANIZATIONS
}

Marcos Pérez-Mendoza $^{1}$ (D) *; María Cruz Cuevas-Álvarez ${ }^{1}$ (D) ; Gerardo Ulises Arias-Moguel ${ }^{1}$.

1. Universidad Juárez Autónoma de Tabasco.

*Correspondencia del Autor: Marcos Pérez-Mendoza, correo electrónico: marcos.perez@ujat.mx.

\section{RESUMEN}

Objetivo: El objetivo del presente trabajo de investigación es diseñar un sistema multimedia para el desarrollo de la competencia intercultural en miembros inmersos en equipos multiculturales dentro de las organizaciones. Material y método: Este trabajo cuantitativo, de tipo descriptivo y de diseño transversal se llevó a cabo con la administración de una encuesta a 100 profesores pertenecientes a una institución de educación superior mexicana, quienes además de trabajar o haber trabajado en empresas internacionales cuentan con una formación intercultural. Resultados: Los datos presentan elementos correspondientes a las tres dimensiones del constructo Competencia Intercultural en los profesores participantes. Este grupo inició su formación de manera virtual la cual reforzaron con exposición a otras culturas de manera presencial. Discusión: Para determinar la efectividad de la plataforma, se requiere de tres fases adicionales: la implementación de la plataforma, seguido por una adecuación a partir de los comentarios de los usuarios y la fase de evaluación de los elementos de la plataforma, así como el desarrollo de la Competencia Intercultural en los usuarios de ésta. Conclusiones: La competencia intercultural permite la minimización o inexistencia de diferencias culturales, la cual podría incrementarse debido al distanciamiento social derivado de la pandemia (SARS COVID19) considerando que la exposición a otras culturas de manera presencial no es posible en la actualidad.

Palabras Clave: Globalización; internacionalización; competencias; competencia intercultural; organizaciones.

Cómo citar:

Pérez-Mendoza, Marcos; Cuevas-Álvarez, María Cruz; Arias-Moguel, Gerardo Ulises. (2021). Diseño de plataforma multimedia para el desarrollo de la competencia intercultural como estrategia de internacionalización en las organizaciones. Revista de Investigaciones Universidad del Quindio, 33(1), 24-36. https://doi.org/10.33975/riuq.vol33n1.446 


\begin{abstract}
Objective: The aim of this research is to design a multimedia system for the development of Intercultural Competence in people immersed in multicultural teams inside organizations. Material and method: This quantitative, descriptive, and cross-sectional research was carried out by administering a survey to 100 faculty members from a Mexican Higher Education Institution, who have been working or have previously worked in international companies and have intercultural training. Results: The data obtained show that participants have elements corresponding to the three dimensions from the Intercultural Competence construct. These faculty members started their training in a virtual mode which was later reinforced with exposition to other cultures in a presence mode. Discussion: In order to determine the effectiveness of the platform, it is required to carry out three additional stages: the implementation of the platform, followed by an adjustment or the necessary changes based on comments or suggestions from the users, and the evaluation stage to the elements of the platform as well as the development of the competence in users. Conclusions: Intercultural competence allows the minimization or non-existence of cultural differences, which could increase due to the social distancing derived from the pandemic (SARS COVID19) considering that exposure to other cultures in a presence mode is no longer possible.
\end{abstract}

Keywords: Globalization; internationalization; competences; intercultural competence; organizations.

\section{INTRODUCCIÓN}

Desde hace varias décadas la multiculturalidad en las empresas se viene gestando por diversas cuestiones: debido a movimientos migratorios, por teletrabajo a través de Internet o por la internacionalización de las empresas, que consiste en crear filiales en otros países o continentes, buscar socios comerciales o socios estratégicos e introducirse en el mercado local de otras naciones.

Al llevar estas acciones a cabo, las empresas asumen que deben tener en sus filas gente de diferentes culturas y países. El trato hacia esa diversidad puede significar la diferencia entre éxito o fracaso. Muchas veces esa diversidad es tratada de manera equivocada pues no se consideran aspectos culturales básicos de cada nacionalidad. Tales como: palabras, expresiones o tonos de voz, por mencionar algunos; considerados códigos distintivos de las culturas, así como la forma de trabajar de quiénes provienen de otros países.

Asimismo, aspectos técnicos y administrativos, como la elaboración de reportes, la organización de juntas corporativas o reuniones de negocio, también son aspectos que requieren un proceso de adaptación; un buen proceso de transculturización laboral puede hacer la diferencia entre el éxito productivo o creación de problemas, en algunos casos considerados detalles mínimos, pero que interfieren con el trabajo diario en una compañía.

A partir de la revisión de la literatura se encuentran los siguientes trabajos relacionados con la competencia intercultural en las organizaciones. En los cuales se menciona la relevancia de que los miembros de equipos multiculturales cuenten con una competencia intercultural que les permita interactuar de manera efectiva y apropiada en diversos ambientes empresariales. 
Tabla 1. Competencia Intercultural en las Organizaciones

\begin{tabular}{ll}
\hline Investigación & Autores \\
\hline El asesor cultural en la empresa & González y López-Cotelo (2015). \\
\hline Defining and understanding organization multicultural competence. & Keršienė y Savanevičiené (2015). \\
\hline La competencia intercultural en las organizaciones: una aproximación teórica & $\begin{array}{l}\text { Elboj, Valero, Iñiguez y Gómez } \\
(2017) .\end{array}$ \\
\hline $\begin{array}{l}\text { Competencias interculturales dentro de la gestión empresarial, realidades de la } \\
\text { logística de comercio exterior en Colombia }\end{array}$ & Correa (2017). \\
\hline La competencia intercultural: habilidad clave para la gestión de las organizaciones. & Cuevas Álvarez et al. (2018). \\
\hline Intercultural competences in slovak business environment & Benčiková, Malá y Ďad’o (2019). \\
\hline
\end{tabular}

Fuente: Elaboración propia

\section{MARCO TEÓRICO}

Dentro de las teorías y modelos encontrados sobre culturas, existen varios precedentes teóricos y de los cuáles se destacan los siguientes trabajos. El modelo de Sensibilidad Intercultural de Bennet (2004) establece que el desarrollo es un proceso lineal de seis etapas:

1. Negación: Aquellos en la etapa de negación no son conscientes de las diferencias culturales. Saben cómo se hacen las cosas en su propia cultura y asumen que en otros lugares las cosas se hacen más o menos igual. Puesto que el individuo en esta etapa asume que las diferencias culturales no existen, es habitual que no muestre interés por la cultura.

2. Defensa: El individuo se da cuenta de algunas diferencias culturales $\mathrm{y}$, a veces, puede sentirse intimidado por ellas. Para aquellos en defensa, el mundo se divide entre "nosotros" y "ellos". Hay dos tipos de defensa: en uno, el individuo ve a "nosotros" como mejores que "ellos; en el otro, llamado defensiva inversa, el individuo admira la otra cultura y ve la propia como inferior.

3. Minimización: El individuo cree que, puesto que todos somos seres humanos, todos somos fundamentalmente lo mismo. En esta etapa, las diferencias culturales parecen triviales, sin importancia: lo que importa es que todos somos seres humanos y que tenemos sentimientos, deseos y necesidades similares.
Este individuo actúa de acuerdo con la norma: "Trata a los demás como te gustaría que te trataran a ti mismo".

4. Aceptación: En esta etapa, el individuo es capaz de reconocer que existen diferencias significantes entre aquellos de diferentes culturas, de modo que acepta y respeta esas diferencias. Sin embargo, en esta etapa, el individuo se siente todavía inseguro acerca de cómo afrontar esas diferencias.

5. Adaptación: El individuo ha ganado la competencia y la experiencia para afrontar las diferencias culturales. Es capaz de adaptar su forma de interactuar y comunicarse con otros, pero también puede adaptarse a los valores que existen en otra cultura. Se siente muy cómodo interactuando con individuos que tienen otros valores o creencias. Ahora ve la cultura como un proceso para relacionarse con otros, no como algo que una persona posee. En esta etapa, el individuo desarrolla empatía cultural y actúa de acuerdo con la norma: "Trata a los demás como les gustaría que les trataran a ellos".

6. La etapa de integración está relacionada con la identidad de uno mismo. En esta etapa, el individuo ha desarrollado un sentido de sí mismo como miembro de dos o más grupos culturales. El individuo se siente cómodo con el concepto de relatividad cultural and es capaz de evaluar la respuesta apropiada en función del contexto en que se encuentra. 
Figura 1. Modelo de Desarrollo de Sensibilidad Intercultural de Bennet (2004).

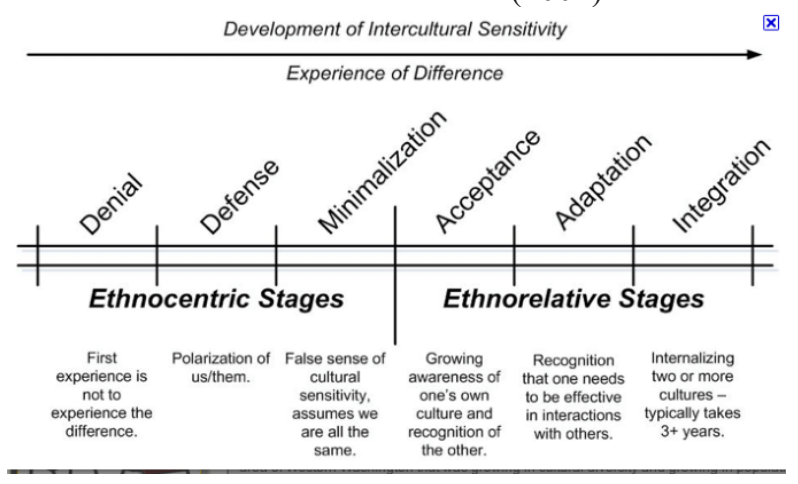

El siguiente modelo encontrado consta de seis dimensiones de la cultura de Hofstede (2011) y se refiere a las dimensiones básicas que la sociedad debe cumplir para organizarse. Permite analizar a las culturas con base en aspectos prioritarios para la comprensión, entendimiento y relación a niveles: profesionales, particulares, familiares y como sociedad.

\section{Individualismo: $\mathrm{El}$ individualismo} es la medida en que las personas se sienten independientes, en lugar de ser interdependientes como miembros de un todo más grande.

2. Distancia de poder: Es la medida en que los miembros menos poderosos de las organizaciones e instituciones, como la familia, aceptan y esperan que el poder se distribuya de manera desigual.

3. Masculinidad/Feminidad: La masculinidad es la medida en que el uso de la fuerza se refrenda socialmente.

4. La evasión de la incertidumbre: Se relaciona con la tolerancia de la sociedad a la incertidumbre y la ambigüedad.

5. Orientación a largo plazo: En una cultura orientada a largo tiempo, la noción básica sobre el mundo es que está en constante cambio, y siempre es necesario prepararse para el futuro. En una cultura orientada a corto tiempo, el mundo es esencialmente como fue creado, de modo que el pasado proporciona una brújula moral y adherirse a él es moralmente bueno.

6. En una cultura indulgente, es bueno ser libre: Hacer lo que tus impulsos quieren que hagas, es bueno. Los amigos son importantes y la vida tiene sentido. En una cultura restringida, la sensación es que la vida es dura, y el deber, no la libertad, es el estado normal del ser.

El último modelo se denomina Competencia Intercultural de Deardoff (2009), el cual se aborda de manera cíclica y recursiva, lo que denota que se puede regresar a una etapa para mejorar o reforzar. La competencia intercultural se define como aquel comportamiento que es efectivo y apropiado en un contexto de comunicación y socialización intercultural (Deardorff, 2011).

En esta competencia es inevitable que no se tengan en cuenta otros procesos cognitivos y actitudinales, como por ejemplo la educación de la persona, las experiencias previas, habilidades o aptitudes, entre otras muchas. Pero adquirir la capacidad de adecuación de un comportamiento a una situación intercultural debe ser el objetivo primordial.

En la Figura 2 se resume, cuáles son las herramientas fundamentales para ser interculturalmente competente. En la mayoría de los casos no se alcanza un punto máximo o un aprendizaje completo en esta ni en otras muchas competencias, todo depende del sujeto, contexto y experiencia se tendrá un comportamiento $\mathrm{u}$ otro.

Figura 2. Modelo de Desarrollo de la Competencia Intercultural de Deardoff (2011).

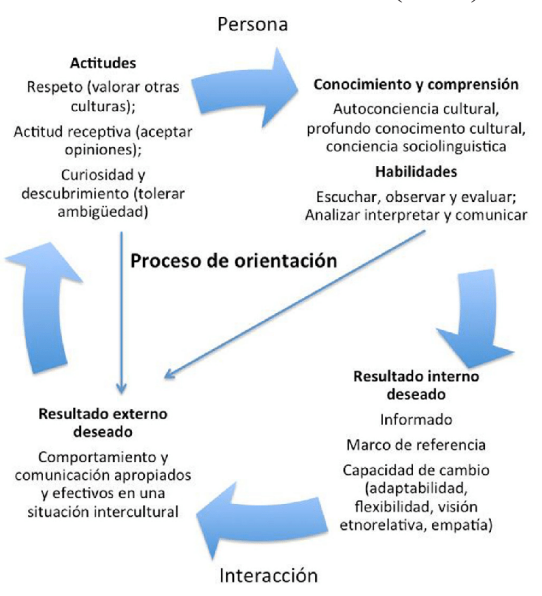




\section{METODOLOGÍA}

El instrumento utilizado fue una encuesta con opciones de respuesta en escala tipo Likert del 1 al 5 siendo 1 totalmente en desacuerdo y 5 totalmente de acuerdo; cada dimensión cuenta con 8 ítems haciendo un total de 24 ítems.

\section{Validez y Confiabilidad del instrumento}

El pilotaje se llevó a cabo con la administración de una encuesta electrónica utilizando Microsoft Forms a 31 participantes, de los cuales se excluyen ocho quedando 24 casos válidos. Se realizó la prueba de confiabilidad de las tres dimensiones con ocho indicadores cada una con el Alfa de Cronbach, obteniendo un .953 porcentaje de confiabilidad.

Figura 3. Confiabilidad del Alfa de Cronbach.

\begin{tabular}{|c|c|}
\hline \multicolumn{2}{|c|}{ Estadísticas de fiabilidad } \\
\hline Alfa de Cronbach & $\mathrm{N}$ de elementos \\
\hline 953 & 24 \\
\hline
\end{tabular}

Los indicadores seleccionados para este instrumento fueron retomados los trabajos de Deardoff (2004), West (2009) y Craft (2010). Los cuales fueron validados a juicio de expertos por un grupo de doctores conformado por cinco personas expertas en competencia intercultural.

\section{Población objetivo:}

Docentes, hombres y mujeres, con capacitación y entrenamiento en estancia académica en la Universidad De Paul, actualmente con ejercicio profesional vigente dentro de las Aulas, Programas y Divisiones Académicas de la UJAT.

\section{Esquema de Muestreo: (Muestreo Aleatorio Simple)}

Se propone un diseño de muestreo probabilístico aleatorio simple, en virtud de que este universo de profesores se comprende solo de 100 docentes y se emplearán métodos electrónicos e interactivos para lograr una cobertura, eficiencia y prontitud mayor a la esperada, aprovechando que estos docentes actualmente se encuentran activos, localizables y con disposición para colaborar con este estudio.

\section{Tasa de no respuesta:}

A partir de la experiencia y encuestas similares realizadas en las entidades, se estima una tasa de no-respuesta menor al $15 \%$.

\section{Tamaño de muestra:}

El tamaño de la muestra se calculó con la siguiente fórmula:

$$
n=\frac{z^{2} N p(1-p)}{e^{2}(N-1)+z^{2} p(1-p)}
$$

Donde $\mathrm{n}$ es el tamaño de la muestra, $\mathrm{N}$ es el tamaño de la población universo, $\mathrm{z}$ es el valor cuantil-normal para el nivel de confianza, e es el margen de y $\mathrm{p}$ es la proporción óptima para estimación de parámetros. De lo anterior, con el $95 \%$ de confianza, bajo el supuesto de un muestreo aleatorio simple y para una proporción de valor 0.5 con un margen de error teórico de $\pm 3 \%$, el diseño descrito proporcionará alrededor de 89 cuestionarios efectivos.

El instrumento logró administrarse al universo conformado por los 100 profesores que realizaron la estancia académica en la Universidad DePaul y que pertenecen a las diversas divisiones de la Universidad Juárez Autónoma de Tabasco. Se contó con 52 participantes femeninos y 48 masculinos. La recolección de datos se realizó del 6 al 31 de enero de 2021 con apoyo de plataformas digitales de educación, redes sociales y comunicación electrónica o telefónica. La encuesta fue aplicada a través del formulario electrónico realizado en Microsoft Forms y distribuido por medio de la aplicación Microsoft Teams, correo electrónico institucional, servicios de mensajería instantánea WhatsApp y Telegram.

Las limitantes presentadas fueron las siguientes: a) poca participación al inicio de la actividad, 
lo cual llevó a requerir apoyo institucional, b) intervención de coordinadores y profesores adscritos a las distintas divisiones que colaboraron en la divulgación del URL del formulario y c) la pandemia y consecuente cuarentena, así como el recién inicio de año con sus festividades alusivas prolongaron el tiempo de respuesta habiendo recibido el día de hoy las últimas encuestas.

\section{RESULTADOS}

De los 100 participantes 39 cuentan con nivel de licenciatura, 4 con especialidad, 26 con maestría y 31 con doctorado (Ver Tabla 3). La mayor participación fue de la división DACEA con 45 participantes, seguido por DACS con 13 y DAEA con 12 participantes, 6 de DACSyH, 5 de DAIS, 4 de DACBIOL, y 3 participantes en cada una de las siguientes divisiones: DACA, DACB, DAIA, DAMC y DAMR (Ver Tabla 4), entre los $21-60$ años (Ver Tabla 5), de los cuales el 48\% estaba conformado por hombres y un $52 \%$ por mujeres (Ver Tabla 6 ).

A partir del análisis estadístico en SPSS de las respuestas obtenidas en la investigación se obtienen los siguientes resultados: La respuesta que más se repitió fue la número 4 (De acuerdo) por el $49 \%$ de los encuestados, seguidos por la número 5 (Totalmente de acuerdo) con un 44\%, y solamente un $7 \%$ seleccionó la número $3(\mathrm{Ni}$ de acuerdo ni en desacuerdo); lo que indica que hay una tendencia positiva hacia la competencia intercultural por parte de los encuestados (Ver Tabla 7). A continuación, se detallan los resultados obtenidos y analizados con SPSS V24 para Mac y clasificados por dimensiones.

\section{Dimensión 1: actitudes.}

Para el caso de la dimensión 1 (actitudes), los encuestados proporcionaron las siguientes respuestas: El 66\% indicó la opción número 4 (De acuerdo), el 26\% prefirió la número 5 (Totalmente de acuerdo), mientras que el $8 \%$ de los encuestados optó por la respuesta número 3 (Ni de acuerdo ni en desacuerdo) (Ver Tabla 8).
Lo anterior nos indica que los participantes gustan estar con personas de otras culturas, aceptar sus opiniones, entender cuando a personas de otras culturas les cuesta trabajo entender nuestras costumbres y creencias; así como respetar los valores, formas de pensar y comportamientos de personas de otras culturas. Por el contrario, solamente un pequeño número de los encuestados cree que las personas de otras culturas son de mente cerrada y que no les agrada que éstas no hablen su idioma.

\section{Dimensión 2: conocimiento.}

Respecto a los resultados de la dimensión 2 (conocimiento), los encuestados contestaron lo siguiente: Un 51\% eligió estar de acuerdo (Respuesta número 4), el 36\% mostró estar totalmente de acuerdo (Respuesta número 5) y el $13 \%$ tuvo una posición neutral al elegir la opción 3 (Ni de acuerdo ni en desacuerdo) en conocer el tipo de temas que se deben abordar al interactuar con otras culturas, así como el volumen de la voz, tono, tipo de acento, ademanes o expresiones que puedan resultar ofensivos para personas de otras culturas (Ver Tabla 9).

Sin embargo, los análisis preliminares en esta dimensiónarrojaronque existeundesconocimiento de los sistemas legales y económicos de otras culturas (36,5\% eligió la opción 3 ni de acuerdo ni en desacuerdo), así como las reglas para expresar conductas apropiadas (no verbal) en las mismas (33.7\% seleccionó la opción 3 ni de acuerdo ni en desacuerdo). De lo anterior se puede inferir también que se haya elegido la opción 3 debido a que existen diversos sistemas legales y un lenguaje corporal (no verbal) diferente en cada país; y que solamente se podría verificar a partir de una entrevista (enfoque cualitativo).

\section{Dimensión 3: habilidades.}

Para la dimensión 3 (habilidades), más de la mitad de los encuestados (59\%) usa pausas y silencios para encajar en situaciones interculturales, observa cuando interactúa con personas de 
distintas culturas, cambia su conducta no verbal, acento y tono cuando la interacción intercultural lo requiere, muestra ser sensible a los mensajes sutiles en estas interacciones y está seguro de que puede socializar y crear contactos futuros para cooperación y colaboración internacional.

Sin embargo, el 2\% de los encuestados muestra poca o nula sensibilidad a los mensajes sutiles y el uso de pausas y silencios para encajar en situaciones interculturales (Ver Tabla 10). Lo anterior también se podría interpretar como una personalidad distraída y que ni en su propia cultura es sensible a mensajes sutiles ni a realizar pausas ni silencios cuando interactúa con otras personas sean de otras culturas o no. Situación particular que podría determinarse a partir de una serie de entrevistas (enfoque cualitativo).

\section{DISCUSIÓN}

Debido a la actual pandemia se requiere de otros métodos o medios para el desarrollo y aprendizaje de la competencia intercultural, donde no existan barreras físicas, económicas ni sanitarias que limiten la adquisición de esta competencia; se comprueba la hipótesis de que la competencia intercultural puede desarrollarse a través de elementos multimedia tal y como lo demostró el grupo de estudio.

A partir de los resultados obtenidos se proponen los siguientes elementos del sistema multimedia para el desarrollo de la competencia intercultural. En la siguiente tabla se enlistan los elementos definidos para el desarrollo de cada una de las dimensiones de la competencia intercultural, siendo $\mathrm{X}$ lo que incluye y refuerza y 0 lo que no aplica.

Tabla 2. Elementos de la estructura y contenidos del sistema multimedia para el desarrollo de la competencia intercultural.

\begin{tabular}{|c|c|c|c|}
\hline Elementos & Actitudes & Conocimiento & Habilidades \\
\hline Vídeos & $\mathrm{X}$ & $\mathrm{X}$ & $\mathrm{X}$ \\
\hline Conversaciones orales y escritas & $\mathrm{X}$ & $\mathrm{X}$ & $\mathrm{X}$ \\
\hline Imágenes y figuras & 0 & $\mathrm{X}$ & $\mathrm{X}$ \\
\hline Ejercicios situacionales & 0 & 0 & $\mathrm{X}$ \\
\hline Ejercicios de reflexión & $\mathrm{X}$ & $\mathrm{X}$ & 0 \\
\hline $\begin{array}{c}\text { Infografías culturales: información } \\
\text { general y protocolos culturales }\end{array}$ & 0 & $\mathrm{X}$ & 0 \\
\hline Infografías geopolítico-económicas & 0 & 0 & $\mathrm{X}$ \\
\hline Salas de interacción virtual & $\mathrm{X}$ & $\mathrm{X}$ & 0 \\
\hline Foros de discusión o blog & $\mathrm{X}$ & $\mathrm{X}$ & $\mathrm{X}$ \\
\hline Autoevaluaciones & $\mathrm{X}$ & & \\
\hline
\end{tabular}

Fuente: Elaboración propia.

La plataforma cuenta con los siguientes materiales precargados: videos cortos relacionados a los gestos y lenguaje corporal en las distintas culturas y en la cultura de los negocios como valor agregado.

Figura 4. Lenguaje corporal como parte de la dimensión de conocimiento

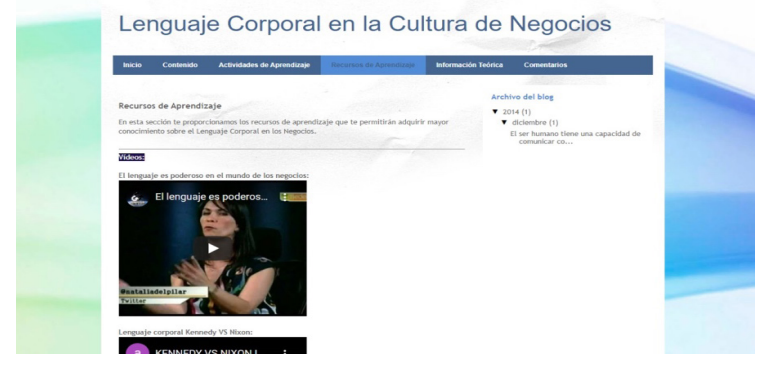


Se cuenta con dos espacios de interacción y discusión: 1) el foro y 2) el blog. En los cuales los usuarios podrán compartir sus impresiones sobre la información provista, así como sus puntos de vista sobre los temas e interactuar con otros usuarios.

Figura 5. Actividades de reforzamiento Lenguaje Corporal en la Cultura de Negocios

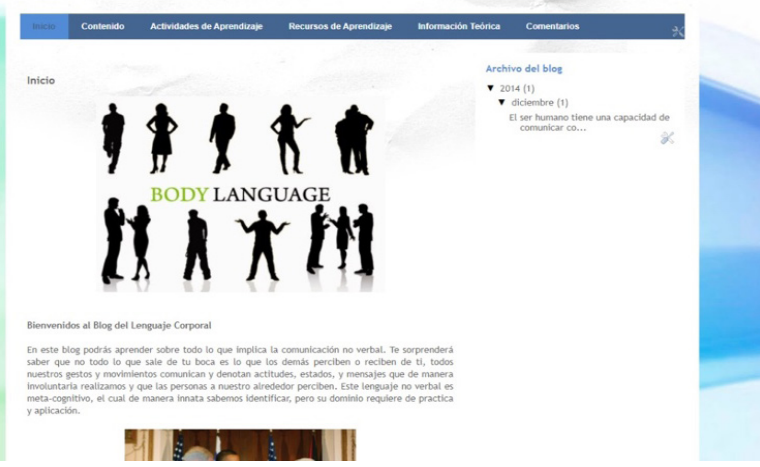

Las actividades de reforzamiento que se diseñaron permitirán la asimilación y el reforzamiento de lo explicado en lecturas, discusión en foros o el área de blog y en los breves videos, a través de la realización de infografías, juegos tipo trivia y actividades de repaso.

Figura 6. Actividades de reforzamiento Lenguaje Corporal en la Cultura de Negocios

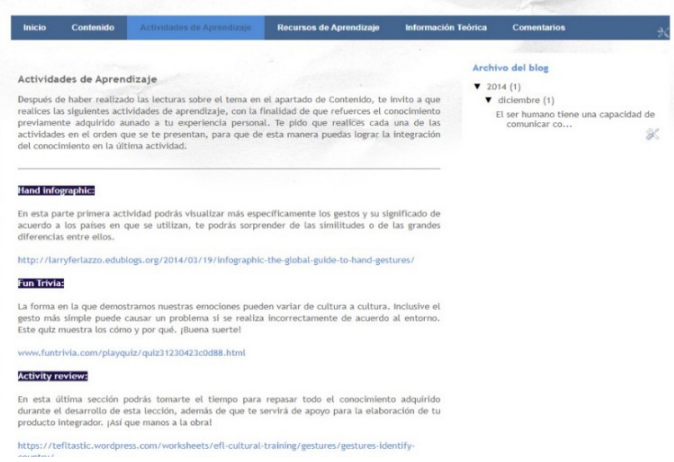

Para corroborar lo aquí propuesto, se requiere de 3 fases adicionales, la primera es la elaboración de la plataforma multimedia que incluya todos los elementos previamente señalados, cumpliendo con las sugerencias de Deardoff (2004) que la competencia intercultural requiere de una serie de actividades para su desarrollo. La segunda fase sería la implementación de esta plataforma con miembros de una organización que estén inmersos en equipos multiculturales. La tercera fase consistiría en la evaluación de los elementos de la plataforma, así como el desarrollo de la competencia intercultural en los usuarios de ésta.

\section{CONCLUSIONES}

La competencia intercultural permite la minimización o inexistencia de diferencias culturales, tal y como lo demostraron los resultados obtenidos a partir de la respuesta de los participantes. Se observaron elementos correspondientes a las tres dimensiones del constructo: actitudes, conocimiento y habilidades, elementos de la Competencia Intercultural.

Este grupo de participantes inició su formación de manera virtual la cual reforzaron de manera presencial durante una estadía en una institución extranjera en tiempos donde no había restricción sanitaria ni distanciamiento social como en la actualidad debido a la pandemia (SARS COVID19) que el mundo está enfrentando. Misma situación que podría ocasionar que las diferencias incrementaran debido a la falta de exposición a otras culturas de manera presencial.

Esta plataforma permitirá la creación de un espacio de aprendizaje intercultural, así como esa exposición necesaria con personas de otras culturas para poner en práctica las actitudes, el conocimiento y las habilidades cada vez requeridas en las organizaciones internacionales para una interacción efectiva entre miembros inmersos en equipos multiculturales. 


\section{REFERENCIAS}

1. Albaum, G., Strandskov, J.; Duerr, E. y Dowd, L. (1994). International Marketing and Export Management. Cambridge: Addison-Wesley.

2. Alonso, J. A. y Donoso, V. (1998): Competir en el exterior. La empresa española y los mercados internacionales. Madrid: ICEX.

3. Benčiková, D., Malá, D. y Ďad'o, J. (2019). Intercultural competences in slovak business environment. E+M. Ekonomie a Management, 22(3), 51-66.

4. Bennett, M. J. (2004). Becoming interculturally competent. In J.S. Wurzel (Ed.) Toward multiculturalism: A reader in multicultural education. Newton, MA: Intercultural Resource Corporation. Recuperado de: https://www.idrinstitute.org/wp-content/uploads/2018/02/becoming_ic_competent.pdf

5. Cavusgil, S. T. (1980). On the Internationalization Process of Firms. European Research, 8(1), 273-281.

6. Correa, M. L. (2017). Competencias interculturales dentro de la gestión empresarial, realidades de la logística de comercio exterior en Colombia. Recuperado de: http://hdl.handle.net/10654/17201.

7. Coviello, N.E. y MCauley, A. (1999). Internationalization and the Smaller Firm: a Review of Contemporary. Management International Review, 39(1), 223-256.

8. Cuevas Álvarez, M. C. (2018). Percepciones del desarrollo de la competencia intercultural en posgrados PNPC de una institución de educación superior mexicana (Tesis Doctoral). Villahermosa: UJAT.

9. Cuevas Álvarez, M. C., Pecero Covarrubias, E., Pérez Mendoza, M., y Macías Olán, C. (2018). La competencia intercultural: habilidad clave para la gestión de las organizaciones. En J. G. I. Gómez, J. E. Sánchez y F. M. Villareal (comp.), La globalización ante los retos de la sustentabilidad, económico-financieros y organizacionales (pp. 67-87). Durango: UJED.

10. Deardoff, D. (2004). The identification and assessment of Intercultural Competence as a student outcome of international education at institutions of Higher Education in the United States. (Tesis doctoral) North Carolina, E. U.: North Carolina State University

11. Deardoff D. (2009). The SAGE handbook of intercultural competence. Los Angeles: SAGE.

12. Durán, J. J. (1994). Factores de competitividad en los procesos de internacionalización de la empresa. Información Comercial Española, 735(1), 21-41.

13. Elboj, C., Valero, D., Iñiguez, T. y Gómez, C. (2017). La competencia intercultural en las organizaciones: una aproximación teórica. Revista Internacional de Organizaciones, 19(1), 75-92

14. Fanjul, E. (2010). Factores culturales e internacionalización de la empresa y la economía. ICE, 856. Recuperado de: https://www.researchgate.net/profile/Enrique_Fanjul/publication/277266752 Factores_culturales_e_internacionalizacion_de_la_empresa/links/55bb8bec08aec0e5f4418c5a/ Factores-culturales-e-internacionalizacion-de-la-empresa.pdf

15. Fanjul, E. (2017). ¿Qué es la globalización? Recuperado de: http://www.iberglobal.com/files/2017/que_es_internacionalizacion-B.pdf

16. Fondo Monetario Internacional [FMI]. (1997). Washington: World Economic Outlook.

17. Forcada, F. J. (1995). Marketing Internacional: Decisiones Estratégicas en el Proceso de Internacionalización Comercial (Tesis Doctoral). Bilbao: Universidad del País Vasco - Euskal HerrikoUnibertsitatea,

18. García, G. (2012). Estrategias de internacionalización de la empresa. Madrid: Pirámide.

19. García, I. (2018). ¿Qué es la internacionalización de las empresas? Recuperado de: https://www. emprendepyme.net/que-es-la-internacionalizacion-de-las-empresas.html

20. García Echevarría, S. (2005). Las adaptaciones organizativas de las empresas para desarrollar su proceso de internacionalización: referencia al caso de la empresa española. Claves de la Economía Mundial ICEX, 1(1), 109-116.

21. Guisado, M. (2003). Estrategia de Multinacionalización de la empresa y Política de Empresa. Madrid, Pirámide.

22. Hofstede, G. (2011). Dimensionalizing Cultures: The Hofstede Model in Context.Online. Readings in Psychology and Culture, 2(1). Recuperado de: https://doi.org/10.9707/2307-0919.1014

23. Hurtado, N. E. (2000). Las Pymes y el Proceso de Internacionalización: Factores Determinantes de su actitud frente a la exportación (Tesis Doctoral). Granada: Universidad De Granada.

24. Keršienè, K., y Savanevičiené, A. (2015). Defining and understanding organization multicultural 
competence. Engineering economics, 42(2), 45-52.

25. Jarillo, J. C. y Martínez, J. (1991). Estrategia Internacional. Más allá de la exportación. Madrid: McGraw-Hill.

26. Johanson, J. y Vahlnne, J. E. (1977). The Internationalization Process of the Firms: a Model of Knowledge Development and Increasing Foreign Market Comminments. Journal of International Business Studies, 8(1), 23-32.

27. Johanson, J. y Wiedersheim-Paul, F. (1975). The Internationalization of the Firm: FourSwedish Cases. Journal of Management Studies, 12(3), 305-322.

28. Levitt, T (1983). La globalización de los mercados. Harvard-Deusto Business Review, 4(1), 49-64.

29. López Duarte, C. (1996). Internacionalización de la empresa española mediante inversión directa en el exterior (Tesis Doctoral). Oviedo: Universidad de Oviedo.

30. López Rodríguez, J. (2004). Análisis de la Actividad Exportadora de la Empresa: una Aproximación desde la Teoría de Recursos y Capacidades (Tesis Doctoral). Coruña: Universidad de Coruña.

31. Luostarinen, R. (1979). The internationalization of the firm. Helsinki: Helsinki School of Economics.

32. Martínez Senra, A. I. (1999). La competitividad como factor clave para la internacionalización de la empresa. Una aplicación a las empresas gallegas del sector del granito (tesis doctoral), Pontevedra: Universidad de Vigo, Vigo.

33. Melin, L. (1992). Internationalization as a Strategy Process. Strategic Management Journal, 13(1), 99-118.

34. Miesenböck, K. J. (1988). Small Business and Exporting: a Literature Review. International Small Business Journal, 6(2), 42-61.

35. Ohmae, K. (1991). El mundo sin fronteras. Ed. Mc Graw-Hill, Madrid

36. Ortega, A. y Espinosa, J. L. (2015). Plan de internacionalización empresarial. Madrid: ESIC.

37. Porter, M. E. (1991). La ventaja competitiva de las naciones. Barcelona: Plaza y Janes.

38. Pla, J. y León, F. (2004). Dirección de Empresas Internacionales. Madrid: Pearson Educación.

39. Rialp, A. (1997). Las fases iniciales del proceso de internacionalización de las empresas industriales catalanas: una aproximación empírica (Tesis Doctoral). Barcelona: Universidad Autónoma de Barcelona.

40. Rialp, A. y Rialp, J. (2005). Las formas actuales de penetración y desarrollo de los mercados internacionales: Caracterización, marcos conceptuales y evidencia empírica en el caso español. Claves de la economía mundial ICEX, 1(1), 99-108.

41. Root, F. R. (1994). Entry Strategies for International Markets. New York: Lexington Books.

42. Velasco, R. (1992). Internacionalización de la Industria Española: Estrategias y Políticas. Economía Industrial, 287(1), 41-51.

43. Villareal, O., Lertxundi, A., Urionabarrenetxea, S. y Matey, J. (2004). Implantación de las empresas vascas en los mercados exteriores: una visión desde la práctica en su operativa diaria. Bilbao: Cluster Conocimiento.

44. Villareal, O. (2005). La internacionalización de la empresa y la empresa multinacional: una revisión conceptual contemporánea. Cuadernos de gestión, 5(2), 55-74.

45. Welch, L. S. y Luostarinen, R. (1988). Internationalization: Evolution of a Concept. Journal of General Management, 14(2), 36-64.

46. Young, S., Hamill, J., Wheeler, C. y Davies, J. R. (1991). Penetración y Desarrollo de los Mercados Internacionales. Barcelona: Estrategias y Gestión, Plaza y Janés.

47. Zaldo, J. M. (1997). Gestión Internacional de las Pymes. Bilbao, SPRI. 


\section{ANEXOS}

Tabla 3 Grados académicos de los participantes.

\begin{tabular}{|c|c|c|c|c|c|}
\hline \multicolumn{6}{|c|}{ Escolaridad } \\
\hline & & Frecuencia & Porcentaje & $\begin{array}{l}\text { Porcentaje } \\
\text { válido }\end{array}$ & $\begin{array}{l}\text { Porcentaje } \\
\text { acumulado }\end{array}$ \\
\hline \multirow[t]{5}{*}{ Válido } & Doctorado & 31 & 31,0 & 31,0 & 31,0 \\
\hline & Especialidad & 4 & 4,0 & 4,0 & 35,0 \\
\hline & Licenciatura & 39 & 39,0 & 39,0 & 74,0 \\
\hline & Maestría & 26 & 26,0 & 26,0 & 100,0 \\
\hline & Total & 100 & 100,0 & 100,0 & \\
\hline
\end{tabular}

Fuente: Elaboración Propia.

Tabla 4. Lugar de adscripción de los participantes. División Académica a la que pertenece.

\begin{tabular}{|c|c|c|c|c|c|}
\hline & & Frecuencia & Porcentaje & $\begin{array}{c}\text { Porcentaje } \\
\text { válido }\end{array}$ & $\begin{array}{l}\text { Porcentaje } \\
\text { acumulado }\end{array}$ \\
\hline \multirow[t]{12}{*}{ Válido } & DACA & 3 & 3,0 & 3,0 & 3,0 \\
\hline & DACB & 3 & 3,0 & 3,0 & 6,0 \\
\hline & DACBIOL & 4 & 4,0 & 4,0 & 10,0 \\
\hline & DACEA & 45 & 45,0 & 45,0 & 55,0 \\
\hline & DACS & 13 & 13,0 & 13,0 & 68,0 \\
\hline & DACSyH & 6 & 6,0 & 6,0 & 74,0 \\
\hline & DAEA & 12 & 12,0 & 12,0 & 86,0 \\
\hline & DAIA & 3 & 3,0 & 3,0 & 89,0 \\
\hline & DAIS & 5 & 5,0 & 5,0 & 94,0 \\
\hline & DAMC & 3 & 3,0 & 3,0 & 97,0 \\
\hline & DAMR & 3 & 3,0 & 3,0 & 100,0 \\
\hline & Total & 100 & 100,0 & 100,0 & \\
\hline
\end{tabular}

Fuente: Elaboración Propia.

Tabla 5. Rango de edades de los participantes

Rango de edad a la que pertenece.

\begin{tabular}{lr|r|r|r|r} 
& Frecuencia & Porcentaje & \multicolumn{1}{c}{$\begin{array}{c}\text { Porcentaje } \\
\text { válido }\end{array}$} & $\begin{array}{l}\text { Porcentaje } \\
\text { acumulado }\end{array}$ \\
\hline Válido & $21-30$ & 36 & 36,0 & 36,0 & 36,0 \\
\cline { 2 - 6 } & $31-40$ & 16 & 16,0 & 16,0 & 52,0 \\
\hline $41-50$ & 24 & 24,0 & 24,0 & 76,0 \\
\hline $51-60$ & 24 & 24,0 & 24,0 & 100,0 \\
\hline
\end{tabular}

Fuente: Elaboración propia.

Tabla 6. Género de los participantes

Género

\begin{tabular}{|c|c|c|c|c|c|}
\hline \\
\hline & & Frecuencia & Porcentaje & $\begin{array}{l}\text { Porcentaje } \\
\text { válido }\end{array}$ & $\begin{array}{l}\text { Porcentaje } \\
\text { acumulado }\end{array}$ \\
\hline \multirow[t]{3}{*}{ Válido } & Femenino & 52 & 52,0 & 52,0 & 52,0 \\
\hline & Masculino & 48 & 48,0 & 48,0 & 100,0 \\
\hline & Total & 100 & 100,0 & 100,0 & \\
\hline
\end{tabular}

Fuente: Elaboración propia.

Tabla 7 Media de frecuencias obtenida de las tres dimensiones.

VTOTALMEDIA (Agrupada)

\begin{tabular}{llrrrr}
\hline \hline & Frecuencia & Porcentaje & $\begin{array}{c}\text { Porcentaje } \\
\text { válido }\end{array}$ & $\begin{array}{c}\text { Porcentaje } \\
\text { acumulado }\end{array}$ \\
\hline Válido & $\begin{array}{l}\text { Ni de acuerdo ni en } \\
\text { desacuerdo }\end{array}$ & 7 & 7,0 & 7,0 & 7,0 \\
& De acuerdo & 49 & 49,0 & 49,0 & 56,0 \\
& Totalmente de acuerdo & 44 & 44,0 & 44,0 & 100,0 \\
& Total & 100 & 100,0 & 100,0 & \\
\hline \hline
\end{tabular}

Fuente: Elaboración propia. 
Tabla 8. Media de frecuencias de la dimensión 1: actitudes.

D1MEDIA (Agrupada)

\begin{tabular}{llrrrr}
\hline \hline & Frecuencia & Porcentaje & $\begin{array}{c}\text { Porcentaje } \\
\text { válido }\end{array}$ & $\begin{array}{l}\text { Porcentaje } \\
\text { acumulado }\end{array}$ \\
\hline Válido & $\begin{array}{l}\text { Ni de acuerdo ni en } \\
\text { desacuerdo }\end{array}$ & 8 & 8,0 & 8,0 & 8,0 \\
& De acuerdo & 66 & 66,0 & 66,0 & 74,0 \\
& Totalmente de acuerdo & 26 & 26,0 & 26,0 & 100,0 \\
& Total & 100 & 100,0 & 100,0 & \\
\hline \hline
\end{tabular}

Fuente: Elaboración propia.

Tabla 9. Media de frecuencias de la dimensión 2: conocimiento.

D2MEDIA (Agrupada)

\begin{tabular}{llrrrr}
\hline \hline & Frecuencia & Porcentaje & $\begin{array}{c}\text { Porcentaje } \\
\text { válido }\end{array}$ & $\begin{array}{c}\text { Porcentaje } \\
\text { acumulado }\end{array}$ \\
\hline Válido & $\begin{array}{l}\text { Ni de acuerdo ni en } \\
\text { desacuerdo }\end{array}$ & 13 & 13,0 & 13,0 & 13,0 \\
& & & & 51,0 & 64,0 \\
& De acuerdo & 51 & 51,0 & 36,0 & 100,0 \\
& Totalmente de acuerdo & 36 & 36,0 & 36,0 & \\
& Total & 100 & 100,0 & 100,0 & \\
\hline \hline
\end{tabular}

Fuente: Elaboración propia.

Tabla 10. Media de frecuencias de la dimensión 3: habilidades.

D3MEDIA (Agrupada)

\begin{tabular}{llrrrr}
\hline \hline & Frecuencia & Porcentaje & $\begin{array}{c}\text { Porcentaje } \\
\text { válido }\end{array}$ & $\begin{array}{c}\text { Porcentaje } \\
\text { acumulado }\end{array}$ \\
\hline Válido & En desacuerdo & 2 & 2,0 & 2,0 & 2,0 \\
& $\begin{array}{l}\text { Ni de acuerdo ni en } \\
\text { desacuerdo }\end{array}$ & 3 & 3,0 & 3,0 & 5,0 \\
& De acuerdo & 36 & 36,0 & 36,0 & 41,0 \\
& Totalmente de acuerdo & 59 & 59,0 & 59,0 & 100,0 \\
& Total & 100 & 100,0 & 100,0 & \\
\hline \hline
\end{tabular}

Fuente: Elaboración propia. 


\section{ÍTEMS E INDICADORES DEL INSTRUMENTO DE RECOLECCIÓN DE DATOS}

\begin{tabular}{|c|c|c|}
\hline & DIMENSIONES & INDICADORES \\
\hline & ACTITUDES & \\
\hline 1 & Me gusta estar con personas de otras culturas & Apertura \\
\hline 2 & Aceptaría las opiniones de personas de otras culturas & Apertura \\
\hline 3 & $\begin{array}{l}\text { Entiendo cuando a personas de otras culturas les cuesta trabajo entender nuestras } \\
\text { costumbres }\end{array}$ & Empatía \\
\hline 4 & Entiendo que a personas de otras culturas les cuesta trabajo entender nuestras creencias & Empatía \\
\hline 5 & Respeto los valores de las personas de diferentes culturas & Respeto \\
\hline 6 & Respeto la forma en que las personas de otras culturas se comportan & Respeto \\
\hline 7 & Creo que las personas de otras culturas son de mente cerrada & Tolerancia \\
\hline \multirow[t]{2}{*}{8} & No me agrada que las personas de otras culturas no hablen mi idioma & Tolerancia \\
\hline & CONOCIMIENTO & \\
\hline 1 & Sé sobre qué tipo de temas abordar cuando interactúo con personas de otras culturas. & información básica \\
\hline 2 & $\begin{array}{l}\text { Sé que para interactuar con personas de otras culturas debo ser interculturalmente } \\
\text { competente }\end{array}$ & información básica \\
\hline 3 & Conozco los sistemas legales y económicos de otras culturas & información específica \\
\hline 4 & Conozco los valores y creencias religiosas de otras culturas & información específica \\
\hline 5 & $\begin{array}{l}\text { La interacción con personas de otras culturas permite crear contactos futuros para } \\
\text { cooperación y colaboración internacional }\end{array}$ & creencias \\
\hline 6 & $\begin{array}{l}\text { Sé que el volumen de la voz, el tono o el tipo de acento puede resultar ofensivo para } \\
\text { personas de otras culturas }\end{array}$ & verbal \\
\hline 7 & $\begin{array}{l}\text { Sé que ciertos ademanes o expresiones pueden resultar ofensivos para otras culturas pero } \\
\text { no en la mía. }\end{array}$ & no verbal \\
\hline \multirow[t]{2}{*}{8} & Conozco las reglas para expresar conductas apropiadas (no verbal) en otras culturas & no verbal \\
\hline & HABILIDADES & \\
\hline 1 & Uso pausas y silencios para encajar en situaciones interculturales & Escucha \\
\hline 2 & Observo mucho cuando interactúo con personas de diferentes culturas & Observa \\
\hline 3 & Cambio mi conducta no verbal cuando la interacción intercultural lo requiere & Observa \\
\hline 4 & Soy sensible a los mensajes sutiles cuando me encuentro en una situación intercultural & Interpreta \\
\hline 5 & $\begin{array}{l}\text { Trato de obtener tanta información como me sea posible cuando interactúo con personas } \\
\text { de diferentes culturas }\end{array}$ & Analiza \\
\hline 6 & Cambio mi conducta verbal (acento y tono) cuando la interacción intercultural lo requiere & Evalúa \\
\hline 7 & Estoy seguro de que puedo socializar con personas de una cultura diferente a la mía & Se relaciona \\
\hline 8 & $\begin{array}{l}\text { Estoy seguro de que puedo crear contactos futuros para cooperación y colaboración } \\
\text { internacional. }\end{array}$ & Se relaciona \\
\hline
\end{tabular}

